\title{
Half-hour global solar radiation forecasting based on static and dynamic multivariate neural networks
}

\author{
Mohammed Ali Jallal*, Samira Chabaa*,** and Abdelouhab Zeroual* \\ *I2SP team, Department of Physics, Faculty of Sciences Semlalia, Cadi Ayyad University, Marrakesh, Morocco \\ **Industrial Engineering Department, National School of Applied Sciences, Ibn Zohr University, Agadir, Morocco \\ Corresponding Author: mohammedali.jallal@edu.uca.ac.ma
}

Submitted: $22 / 07 / 2019$

Revised: $\quad 02 / 02 / 2020$

Accepted: $12 / 05 / 2020$

\begin{abstract}
Precise global solar radiation (GSR) measurements in a given location are very essential for designing and supervising solar energy systems. In the case of rarity or absence of these measurements, it is important to have a theoretical or empirical model to compute the GSR values. Therefore, the main goal of this work is to offer, to designers and engineers of solar energy systems, an appropriate and accurate way to predict the half-hour global solar radiation (HHGSR) time series from some available meteorological parameters (relative humidity, air temperature, wind speed, precipitation, and acquisition time vector in half-hour scale). For that purpose, two intelligent models are developed: the first one is a multivariate dynamic neural network with feedback connection, and the second is a multivariate static neural network. The database used to build these models was recorded in Agdal's meteorological station in Marrakesh, Morocco, during the years of 2013 and 2014, and it was divided into two subsets. The first subset is used for training and validating the models, and the second subset is used for testing the efficiency and the robustness of the developed models. The obtained results, in terms of the statistical performance indicators, demonstrate the efficiency of the developed forecasting models to accurately predict the HHGSR parameter in the city of Marrakesh, Morocco.
\end{abstract}

Keywords: Global Solar Radiation; Static Neural Network; Dynamic Neural Network; Artificial Neural Networks; Multivariate; Prediction.

\section{INTRODUCTION}

Solar energy is one of the most important sources of available energy. Hence, accurate knowledge of solar radiation distribution at a given geographical location is extremely important for the development of solar energy systems and for the evaluation of their performance. Unfortunately, for many developing countries, solar radiation measurements are not readily available. Therefore, it is important to develop models for estimating the solar radiation measurements based on available geographical, astronomical, and meteorological data.

Morocco is an excellent land of sun, where it is among the countries having one of the uppermost rates of solar insulation (SI), around 3000 hours per year of sunshine, but up to 3600 hours in the desert. This climate is very favorable for the exploitation of solar energy in different areas, but the distribution of solar radiation is almost unknown. Indeed, global solar radiation (GSR) values are the most important input for solar energy applications. Certainly, the setting up of a solar project installation confronts problems related to the scarceness or even the absence of the global solar radiation measurements (Chiteka et al., 2016). To overcome these problems, several models have been developed in the literature for various locations in the world in order to estimate the GSR series, with artificial neural networks being among them. 
Alsina et al. (2016) have predicted the monthly GSR over Italy via artificial neural networks (ANNs) by using a set of climatological parameters, including the geographical coordinates. Chiteka el al. (2016) have applied the ANN technique for predicting global horizontal irradiation (GHI) for major locations in Zimbabwe using geographical data and some meteorological parameters. Gani et al. (2016) have adopted the autoregressive ANN with exogenous inputs (NN-ARX) and the adaptive neuro-fuzzy inference system (ANFIS) in order to predict the daily horizontal global solar radiation using the day of the year as a single input. Gutierrez-Corea et al. (2016) have applied the ANN technique in the short-term prediction of global solar irradiance (GSI) by using a new methodology based on different meteorological variables observations recorded in parallel by neighboring sensors. Shaddel et al. (2016) have estimated the hourly GSI parameter for tilted planes via ANNs.

In many of the studies analyzed for GSR time series prediction, the following gaps were found:

(1) the amount of data used by the most studies is not enough to create robust and accurate predictive models that can handle the chaotic behavior of the climatic conditions; (2) the studied time series in the previous works presented simple nonlinearity and slow variability that can be modeled using just some statistical linear methods; (3) some of the contributions have focused on the forecasting methods that use only the endogenous patterns to perform the GSR prediction, but the accuracy was not improved.

In the previous related works, the authors have developed several predictive models of GSR based mainly on statistical methods. In 1995, Zeroual et al. have proposed two stochastic models developed using daily global solar radiation measured over three years on a horizontal surface in Marrakesh, Morocco. In 1996, the same authors have studied the application of the diffuse-global correlation to estimate solar radiation on tilted surfaces in Marrakesh, Morocco (Zeroual et al., 1996). Thereafter, in 2002, they have developed a moving average system identification using higher-order cumulants applied to model solar radiation (Safi et al., 2002).

The authors have also developed models based on artificial intelligence techniques to predict the GSR data. In 2007, a new technique based on Takagi-Sugeno fuzzy systems is proposed to predict the daily GSR in Marrakesh, Morocco (Iqdour et al., 2007a). In the same year, Iqdour et al. have used the MLP neural networks trained by the Polack-Ribière algorithm for daily GSR estimation. Then, in 2013, El Badaoui et al. have applied multivariate MLP neural networks for predicting daily GSR in the region of Sebt El Guerdane, Agadir, Morocco, from several meteorological parameters that are measured during five years.

In the present work, the prediction of the ahead half-hour global solar radiation (HHGSR) was performed by using two intelligent models based on the multilayer ANNs in order to overcome the presented gaps of the aforementioned approaches. The first model is a multivariate dynamic neural network with feedback connection (M1), and the second model is a multivariate static neural network (M2). Five independent inputs (relative humidity $(r H)$, air temperature $(a T)$, wind speed $(W S)$, precipitation $(P r)$, and acquisition time vector in half-hour scale $(t)$ ), measured in Agdal, Marrakesh, Morocco, during the years of 2013 and 2014, were used to predict half-hour global solar radiation (HHGSR) time series. Among the developed models, an accurate ANN model was selected based on different statistical metrics.

In this study, the essential elements of innovation and interest for the scientific community and the practitioners are used to improve the HHGSR prediction accuracy ahead. The knowledge of the HHGSR data in advance allows solar system engineers to do a set of procedures for scheduling solar power plants production and monitoring their performance in the case of sensor damage. The prediction was performed by using the most relevant independent parameters measured through a sensitivity analysis, and then the dynamic and the statistic approaches were adopted to achieve this goal.

\section{ARTIFICIAL NEURAL NETWORKS}

\section{Artificial neural networks architecture}

ANN is a three-layer computing system, input, output, and intermediate hidden layers, with their corresponding neurons. The neurons of each layer are connected to the next layer's neurons, which lead to a huge number of 
connections, and each connection has a weight associated with it. The hidden layer learns to infer the relationship existing between the input and the output variables.

The output of each neuron is calculated by applying an activation function to the weighted sum of the inputs that have been received by the neuron (Fig. 1) (El Badaoui et al., 2013).

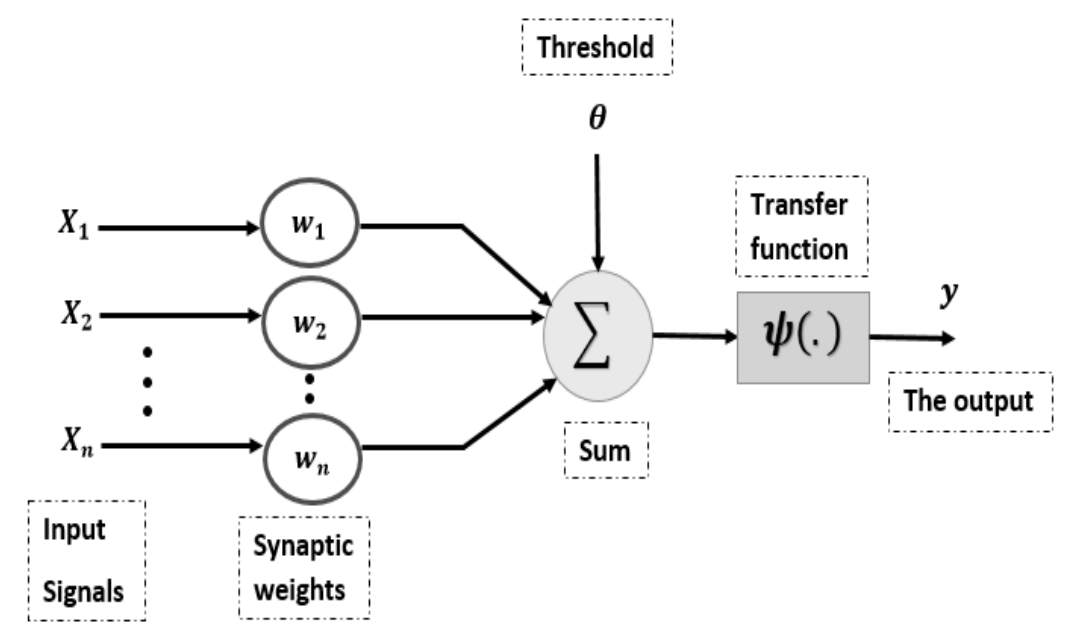

Figure 1. Artificial neuron structure.

An artificial neuron is considered as an element of information processing. It receives external inputs $\left(x_{1}, x_{2}, \ldots, x_{n}\right)$ and produces a result $y$ at the output (Iqdour et al., 2007; Chabaa et al., 2010; Mohandes et al., 1998):

$$
\begin{aligned}
& y=\psi(u(w, x)) \\
& u(w, x)=\sum_{j=1}^{n} w_{j} x_{j}+\theta=W^{\prime} X+\theta
\end{aligned}
$$

where $w_{1}, w_{2}, \ldots, w_{n}$ are the synaptic weights associated with each connection, $W=\left[w_{1}, w_{2}, \ldots, w_{n}\right]^{t}$ is the vector of synaptic weights, $X=\left(x_{1}, x_{2}, \ldots, x_{n}\right)$ is the vector of inputs, and $\theta$ is the bias or threshold vector.

$\psi$ is the nonlinear activation function. Various activation function types can be used, with logistic sigmoid function (LS), hyperbolic tangent sigmoid function (TS), Gaussian radial basic function (RGB), and linear activation function (L) (Assi 2011) being among them, where the choice of the activation function type depends on the application.

\section{Training neural networks}

To create a predictive neural model, a supervised learning approach is adopted. The network should adjust its weights to reduce the difference between the desired response and the output of the network. This procedure is applied repeatedly until the defined conditions are satisfied.

There are various backpropagation algorithms to train ANN forecasting models, but the efficient and the robust one is the Levenberg-Marquardt (LM) algorithm (F. Li et al., 2018; Chabaa et al., 2010; Saha et al., 2018; Prabhaker et al., 2012; Bear et al., 1995). The LM algorithm, like Quasi-Newton methods, was designed to approach secondorder training speed without the need to compute the Hessian matrix (Chabaa et al., 2010; Muhammad et al., 2008). The performance function, $f_{p}(w)$, of LM algorithm has the form of the error vector sum of squares, which is defined by the following equation:

$$
f_{p}(w)=\frac{1}{2} e^{T}(w) e(w)=\frac{1}{2} \sum_{i=1}^{k} \sum_{j=1}^{p}\left(O_{i j}-t_{i j}\right)^{2}
$$


where $W=\left[w_{1}, w_{2}, \ldots, w_{n}\right]^{t}$ is the vector of all the network's weights. $O_{i j}, t_{i j}, i$ and $j$ are, respectively, the observed output, the desired output, the iteration number, and the number of samples.

Newton's method, for minimizing an objective function, is applied to update the weight values using the following formula:

$w_{i+1}=w_{i}-H^{-1} \nabla f_{p}(w)$

where $\nabla f_{p}(w)$ is the gradient of $f_{p}(w)$, and $\mathrm{H}$ is the Hessian matrix approximated by

$$
H \approx J^{T} J
$$

The following equation computes the gradient:

$$
\nabla f_{p}(w) \approx J^{T} e
$$

The Jacobian matrix $J$ contains the first derivatives of the vector of network errors $e=\left(e_{11}, e_{12}, \ldots, e_{1 k}, \ldots, e_{p 1}\right.$, $\left.e_{p 2}, \ldots, e_{p k}\right)^{T}$ with respect to the weights and the biases.

The Jacobian matrix is computed as follows:

$$
J=\left[\begin{array}{cccc}
\frac{\partial e_{11}}{\partial w_{1}} & \frac{\partial e_{11}}{\partial w_{2}} & \ldots & \frac{\partial e_{11}}{\partial w_{n}} \\
\frac{\partial e_{12}}{\partial w_{1}} & \frac{\partial e_{12}}{\partial w_{2}} & \ldots & \frac{\partial e_{12}}{\partial w_{n}} \\
\vdots & \vdots & \ldots & \vdots \\
\frac{\partial e_{1 k}}{\partial w_{1}} & \frac{\partial e_{1 k}}{\partial w_{2}} & \ldots & \frac{\partial e_{k 1}}{\partial w_{n}} \\
\vdots & \vdots & \ldots .: & \vdots \\
\frac{\partial e_{p 1}}{\partial w_{1}} & \frac{\partial e_{p 1}}{\partial w_{2}} & \ldots & \frac{\partial e_{p 1}}{\partial w_{n}} \\
\frac{\partial e_{p 2}}{\partial w_{1}} & \frac{\partial e_{p 2}}{\partial w_{2}} & \ldots & \frac{\partial e_{p 2}}{\partial w_{n}} \\
\vdots & \vdots & & \vdots \\
\frac{\partial e_{p k}}{\partial w_{1}} & \frac{\partial e_{p k}}{\partial w_{2}} & \ldots & \frac{\partial e_{p k}}{\partial w_{n}}
\end{array}\right]
$$

Besides, the Gauss-Newton update formula can be calculated by

$$
w_{i+1}=w_{i}-\left(J_{i}^{T} J_{i}\right)^{-1} J_{i}^{T} e_{i}
$$

where $\left(J^{T} J\right)$ is a positive definite matrix, but if it is not, some perturbations are made into it, to control the probability of being a nonpositive definite matrix (Chabaa et al., 2010; Muhammad et al., 2008). The Hessian matrix, in this case, becomes as follows:

$$
H \approx J^{T} J+\mu I
$$

Consequently,

$$
w_{i+1}=w_{i}-\left(J_{i}^{T} J_{i}+\mu I\right)^{-1} J_{i}^{T} e_{i}
$$


where the scalar $\mu$ is called the learning parameter that controls the descent step of the algorithm, and its value during the training process needs to be decreased by the iterative approach to reach a minimum (Muhammad et al., 2008; Min et al., 2017).

\section{Static and dynamic neural networks}

\section{Static neural network}

In the static neural network, the information runs from the inputs to the outputs without any feedbacks (Fig. 2) (Dewa et al., 2017; Kocadagli et al., 2017; Jallal et al., 2019).

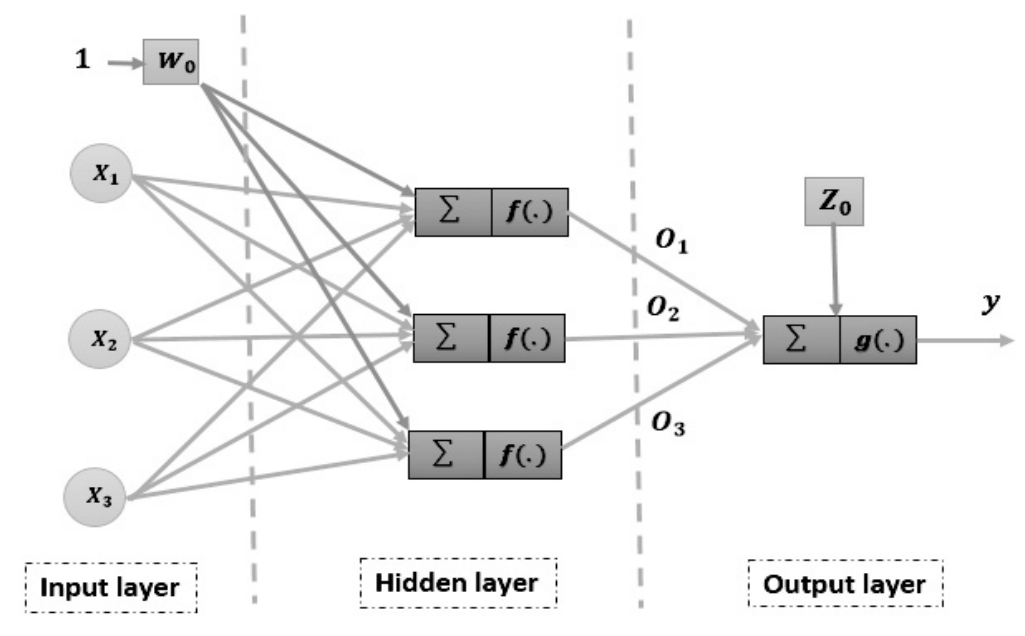

Figure 2. Static neural network.

The output $y$ of the static network is expressed as (Iqdour et al., 2007; El Badaoui et al., 2013; Le et al., 2014)

$y=g\left(W_{2 i} o_{i}+Z_{0}\right)$

where $o_{i}$ is the output of neuron $i$ in the hidden layer, defined by

$o_{i}=f\left(W_{1 i} X+\theta_{i}\right)$

$f$ and $g$ are the activation functions of the hidden layer and output layer, respectively. $X$ is the vector of the inputs, $W_{1 \mathrm{i}}$ is the matrix of connection's weights linking the input layer to the hidden layer, $\theta$ is the bias vector of the neurons in the hidden layer, $W_{2 \mathrm{i}}$ is the matrix of the weights of connections linking the hidden layer to the output layer, and $z_{0}$ is the bias of the output neuron.

\section{Dynamic neural network with feedback connection}

A dynamic network with feedback connection (DNFC), governed by one or more differential equations, results from the composition of the functions performed by each of the neurons and the time delay (TDL) associated with each of the connections (sheng Li et al., 2017; Bonilla et al., 2017).

The architecture of the dynamic neural network with a feedback connection is described by the representation in Fig. 3. 


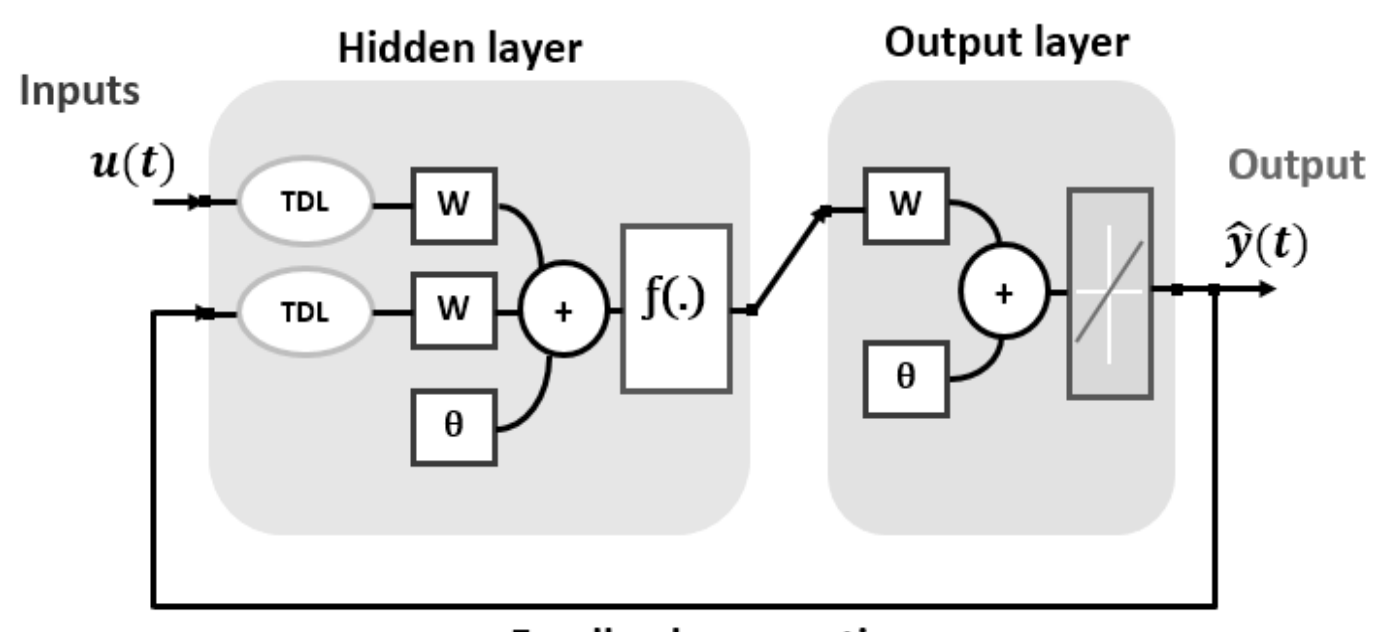

Feedback connection

Figure 3. Architecture of the dynamic neural network with feedback connection.

The DNFC model is based on the autoregressive process with exogenous inputs (ARX), commonly used in time series modeling (Gani et al., 2016; Cardona et al., 2017; Rzadkowski et al., 2015). The output $y(t)$ of the DNFC is given by the following equation:

$$
y(t)=f\left(\hat{y}(t-1), \hat{y}(t-2), \ldots, \hat{y}\left(t-n_{y}\right), u(t-1), u(t-2), \ldots, u\left(t-n_{u}\right)\right)
$$

where the next value of the dependent output signal $y(t)$ is regressed on the previous values of the predicted output signal $\hat{y}(t)$ and independent exogenous input signal $u(t)$.

\section{DATABASE DESCRIPTION AND ANALYSIS}

In this study, the data used were measured every half an hour from sunset to sunrise for 2 years $(2013,2014)$ at Agdal's station in Marrakesh, Morocco (latitude $31^{\circ} 37^{\prime} \mathrm{N}$, longitude $08^{\circ} 02^{\prime} \mathrm{W}$, elevation $463 \mathrm{~m}$ ). Marrakesh region is characterized by a semiarid climate and an extremely high rate of solar radiation, which can be harnessed to produce clean energy from solar source.

To create the static and dynamic neural networks, respectively, M2 and M1 models, the database was divided into two subsets. The measurements corresponding to the year 2013 are used to train and validate the models (Phase I), whereas the data measured during 2014 are saved for testing the effectiveness and the robustness of the models (Phase II).

In order to minimize the training time and to ensure the homogenization of the values propagated in the network, the data used in Phases I and II are normalized between -1 and 1 . This normalization adapted the data to the requirements of the transfer function used by the neural network, with respect to their minimum and maximum values. The normalization is achieved by the following equation (Jallal el al. 2019b):

$$
\bar{y}_{i}=\frac{2\left(y_{i}-y_{i(\min )}\right)}{\left(y_{i(\max )}-y_{i(\min )}\right)}-1
$$




\section{DEVELOPMENT OF STATIC AND DYNAMIC MODELS}

To define the optimal structure for both models, we used the multivariate dynamic neural model with feedback connection (M1) and the multivariate static neural model (M2). The number of hidden layers and the number of neurons in each hidden layer need to be changed wisely. Consequently, different combinations of activation functions in hidden and output layers have to be tested.

The optimal structure corresponds to minimum values in the two phases (Phases I and II) of all the following statistical metrics (Jachner et al., 2007):

Centered Mean Squared Error (CMSE):

$$
C M S E=\frac{1}{N-1} \sum_{i=1}^{N}\left(H H G S R_{i}-H \widehat{H G S} R_{i}-\operatorname{mean}\left(H H G S R_{i}-H \widehat{H G S} R_{i}\right)\right)^{2}
$$

Centered Mean Absolute Error (CMAE):

$$
C M A E=\frac{1}{N} \sum_{i=1}^{N}\left|H H G S R_{i}-H \widehat{H G S} R_{i}-\operatorname{median}\left(H H G S R_{i}-H \widehat{H G S} R_{i}\right)\right|
$$

where the predicted and the measured time series of the HHGSR parameter were centered in advance by removing the mean value in order to remove the mean discrepancy.

Moreover, the optimal structure is the one that maximizes the value of the correlation coefficient $R$, which is defined by Eq. (16) to reach the value 1 (Jallal el al. 2019c).

$$
R=\sqrt{\sum_{i=1}^{N}\left(H \overline{H G S} R_{i}-\overline{H H G S R}\right)^{2} / \sum_{i=1}^{N}\left(H H G S R_{i}-\overline{H H G S R}\right)^{2}}
$$

where

$\mathrm{N}$ : Number of input/output patterns,

$H_{H G S R}$ : Measured half-hour global solar radiation time series,

$H \widehat{H G S} R_{i}$ : Predicted half-hour global solar radiation time series,

$\overline{H H G S R}$ : Mean value of the measured half-hour global solar radiation time series.

To apply the M1 and the M2 models for predicting HHGSR at the instant $t+1$, five input parameters (relative humidity $r H(t)$, air temperature $a T(t)$, wind speed $W s(t)$, precipitation $\operatorname{Pr}(t)$, and acquisition time vector in half-hour scale $t$ ) are used as shown in Figure 4, respectively. 


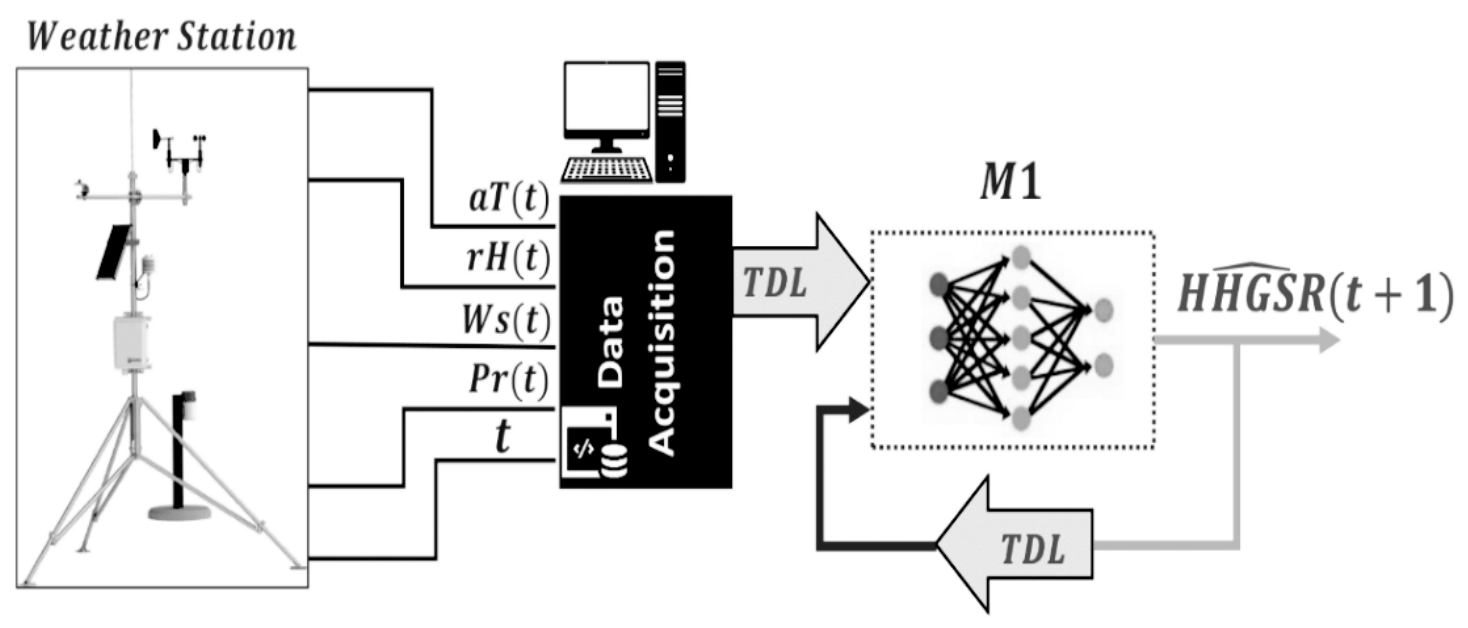

(a)

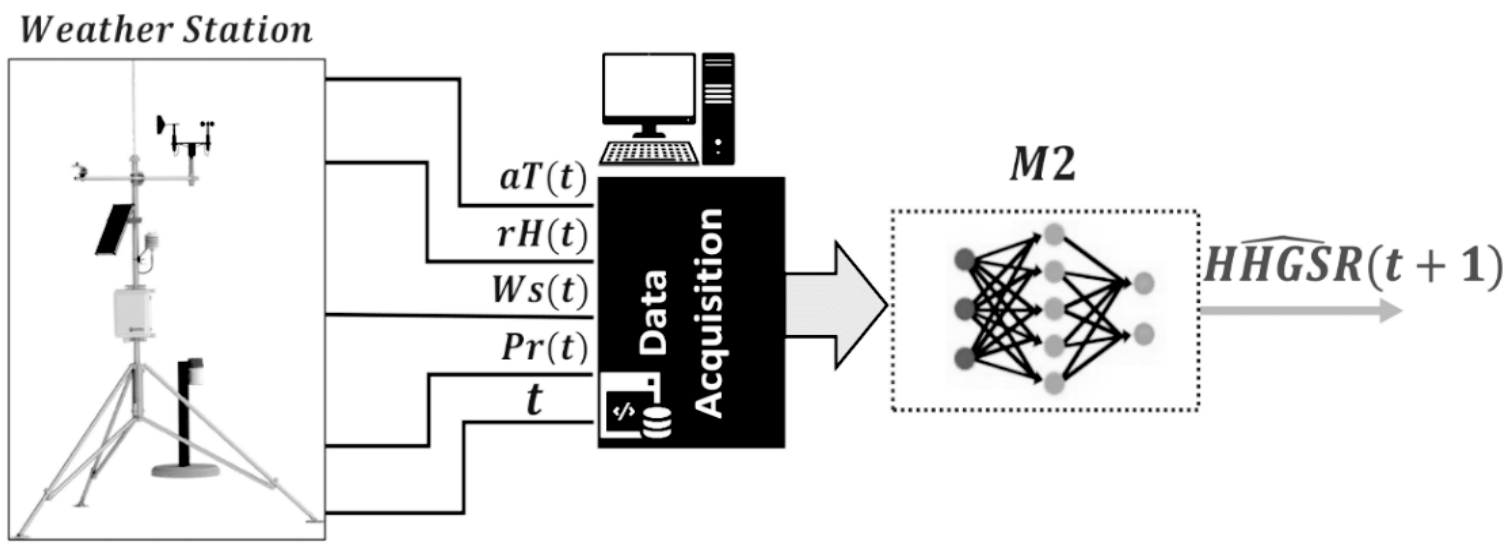

(b)

Figure 4. ANNs architectures: (a) M1 model architecture; (b) M2 model architecture.

\section{RESULTS AND DISCUSSION}

\section{Relevance analysis of the input variables}

This study aimed to predict HHGSR measurements from other independent variables $(W s, a T, r H, P r, t)$.

To improve the performances of both model M1 and M2 and to understand the relevance of each input parameter, the relevance determination test (RDT) is implemented. This test generates a list of diverse scenarios $\{S 1, S 2, S 3, \ldots, S 7\}$ that showed their relevance for both models. Figure 5 plots the HHGSR centered mean squared errors for the models M1 and M2 as a function of the generated scenarios. For each scenario, the considered inputs are marked by a blue circle.

The examination of the results shows that the diminution of the input number reduces the performance of both models. The obtained result of the RDT proves that the best input scenario is $\mathrm{S} 1$, which used five inputs $(W s, a T, r H, P r, t)$. This scenario leads to minimize the CMSE statistical indicator, compared to the others $\{S 2, S 3, \ldots, S 7\}$. This input scenario is selected as the final one for both models. More details about the models M1 and M2 construction are presented in the following section. 


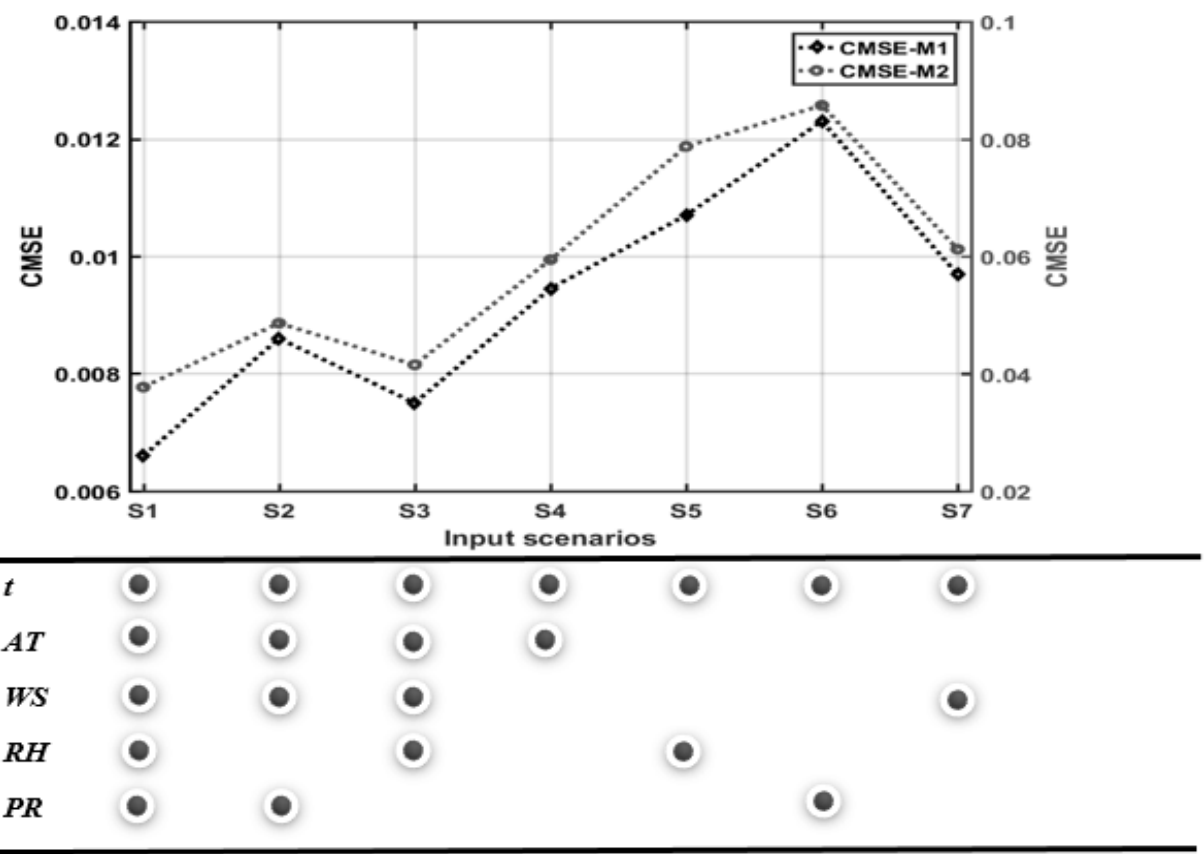

Figure 5. Performance of the models $M 1$ and $M 2$ varying the input variables.

\section{Autoregressive orders of the M1 model determination}

To determine the M1 model structure, the appropriate orders of the autoregressive terms of the direct and feedback inputs need to be determined. To solve this problem, the order of these terms has been varied between 1 and 5 , and the best combination of orders is the one that minimizes the statistical indicators CMSE and CMAE in the testing phase. In Figure 6, the combinations of the autoregressive orders as a function of the statistical indicators (CMSE, CMAE) in the testing phase are depicted.

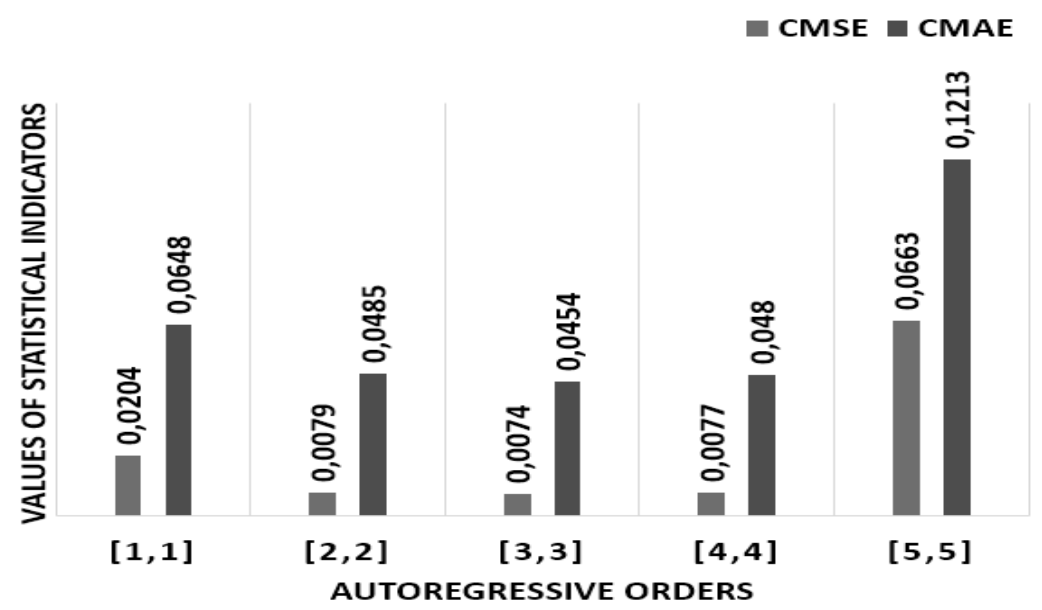

Figure 6. Autoregressive orders for direct and feedback inputs vs statistical indicators.

The obtained values of the CMSE and CMAE demonstrate that the best choice is a delay vector of order 3 for the direct and feedback inputs. 


\section{Building of M1 and M2 models}

The determination of the hidden layer number (step 1), the number of neurons in each hidden layer (step 2), and the corresponding activation function for each layer (step 3) are necessary to build M1 and M2 models. Therefore, different combinations are tested for steps 1 and 2. The obtained results, for step 1, using the statistical indicator CMSE in the testing phases, are presented in Figure 7.

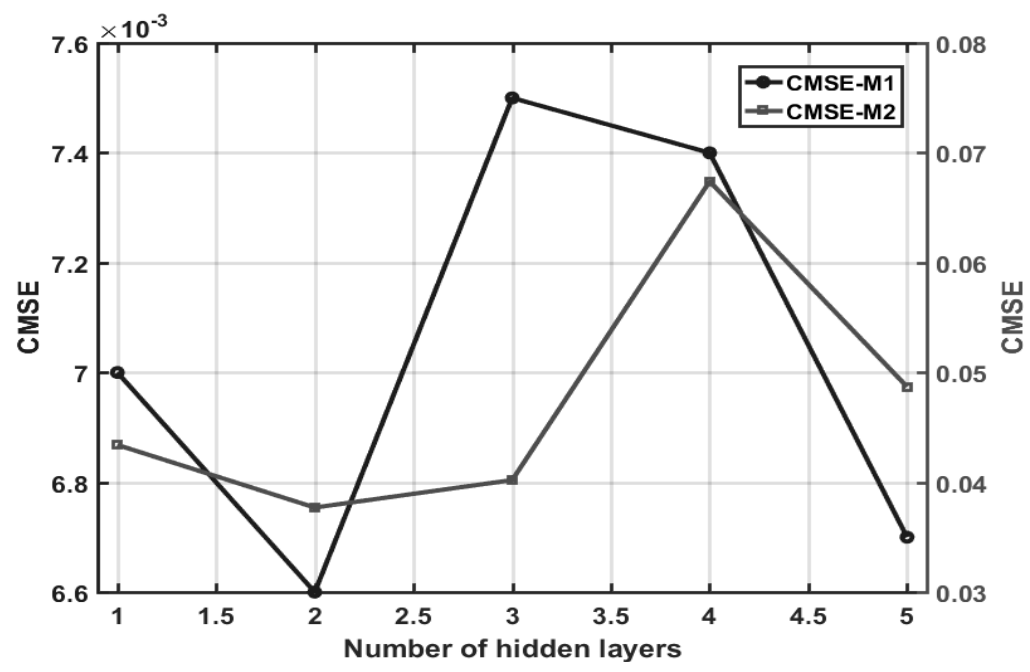

Figure 7. Number of hidden layers vs CMSE for models M1 and M2.

These results demonstrate the pertinence of the choice of two hidden layers for both models. In addition, the increase in the hidden layers number affects the computing time without a significant gain in the performance for both models.

For step 2, in Figure 8, the variation of the number of neurons in the hidden layers between one and 26 for each hidden layer is depicted. This variation indicates the advantages of the structures $\{8-4\}$ for the M1 model and $\{25-10\}$ for the M2 model, which is proved by the values closer to zero for the statistical indicator CMSE.

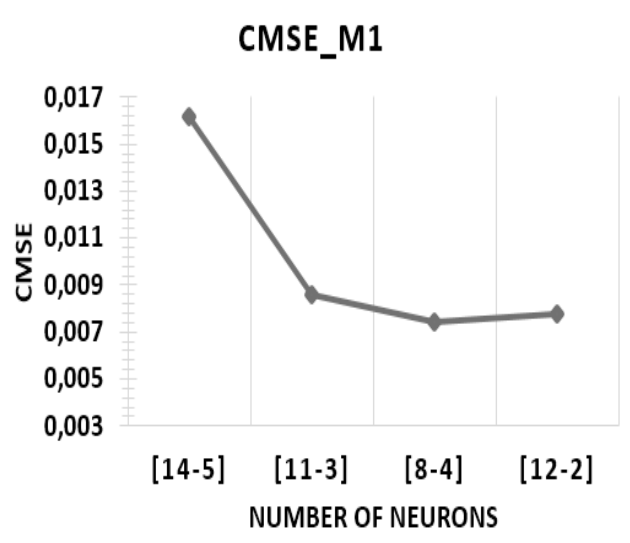

(a)

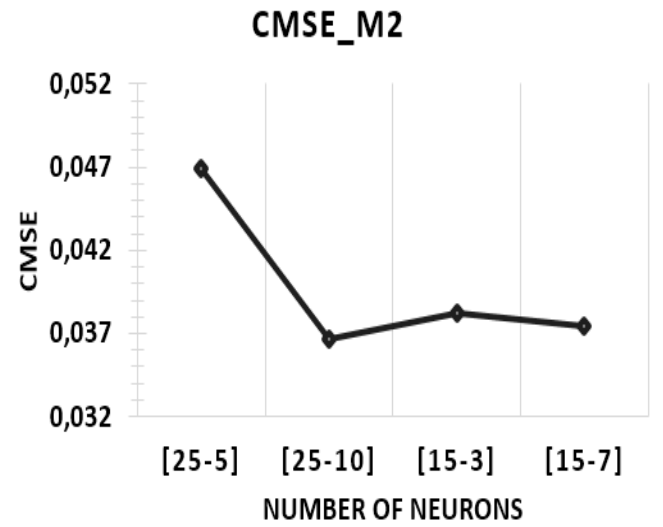

(b)

Figure 8. Number of neurons in the hidden layers vs CMSE for (a) M1 and (b) M2 models.

The few numbers of neurons obtained in the hidden layers of the M1 model structure can significantly reduce the numbers of computing operations in the network and consequently minimize the training time. According to the attained values of the statistical criteria, the M1 model is more efficient than the M2 model. 
Afterwards, to find out the corresponding activation function for each layer (step 3), in Figure 9, different combinations in hidden and output layers were tested.

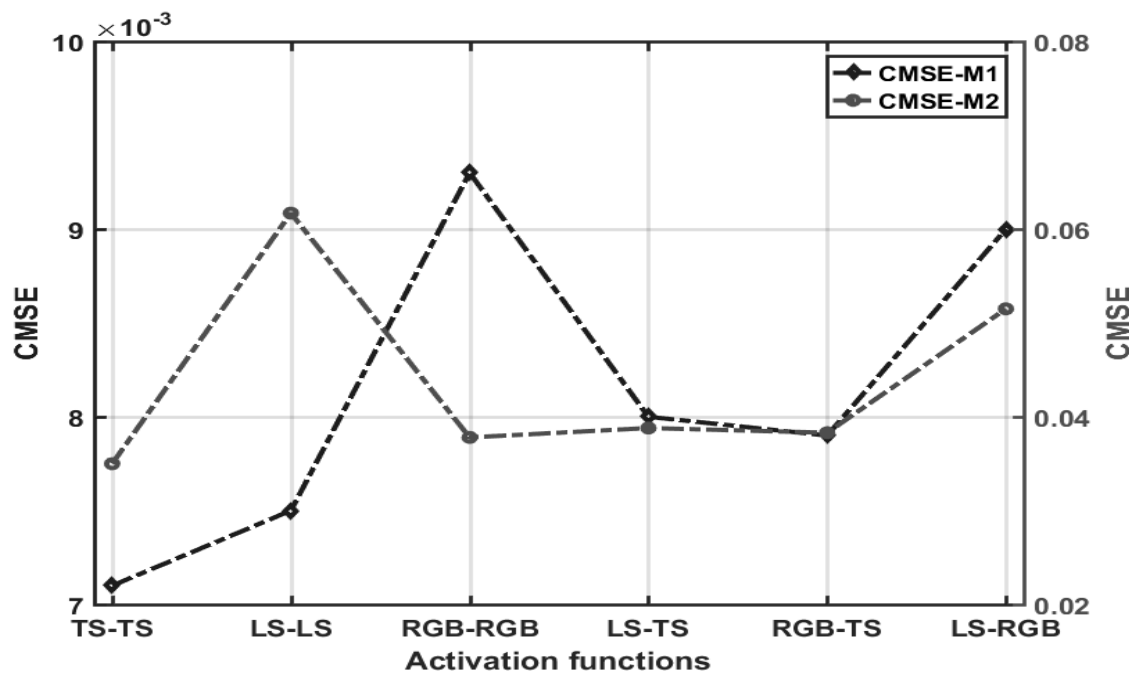

Figure 9. Activation functions in each hidden layer for models M1 and M2.

The results show that the best combination of the activation functions in the hidden and the output layers is TSTS-L for both models.

\section{Synthesis study}

From all the previous results, the dynamic model (M1) gives more precision in terms of the adopted statistical criteria than the static model (M2) (Fig. 10). Moreover, M1 model demonstrates a high accuracy level in terms of prediction quality compared to the different literature contributions (Loutfi et al., 2017; Ahmad et al., 2015). In addition, to demonstrate the efficiency of the developed models based on the LM training algorithms, in Figure 11, the evolutions of measured and predicted HHGSR time series corresponding to the two models in the testing phase for 500 samples are presented. From the evolutions, a slight overestimation in the winter season during the testing phase for the M2 model was recorded. This weakness may be due to the rapid fluctuations normally observed in these different meteorological parameters during this season.

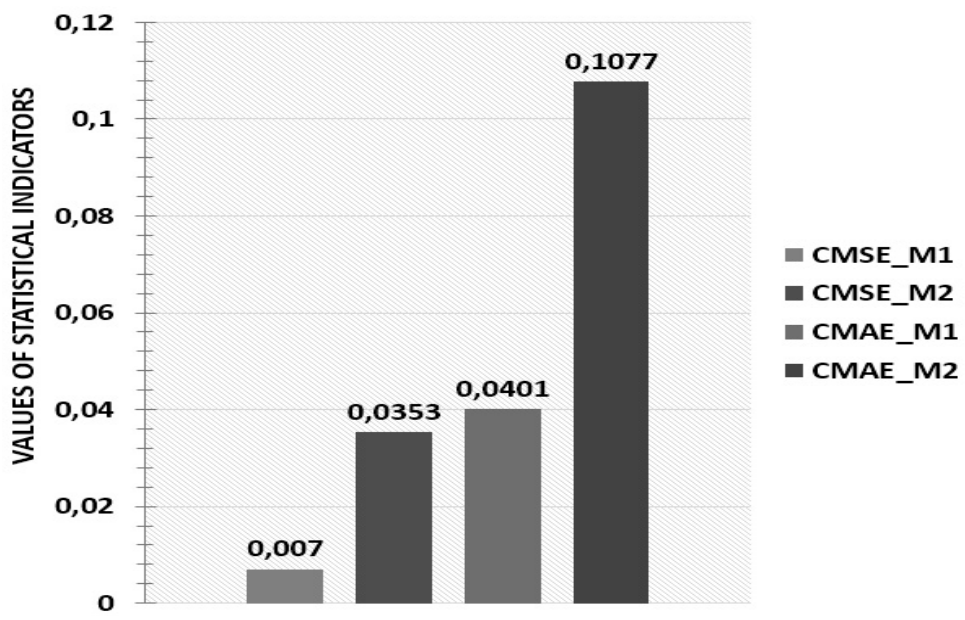

Figure 10. Statistical discrepancies of M1 and M2 models. 


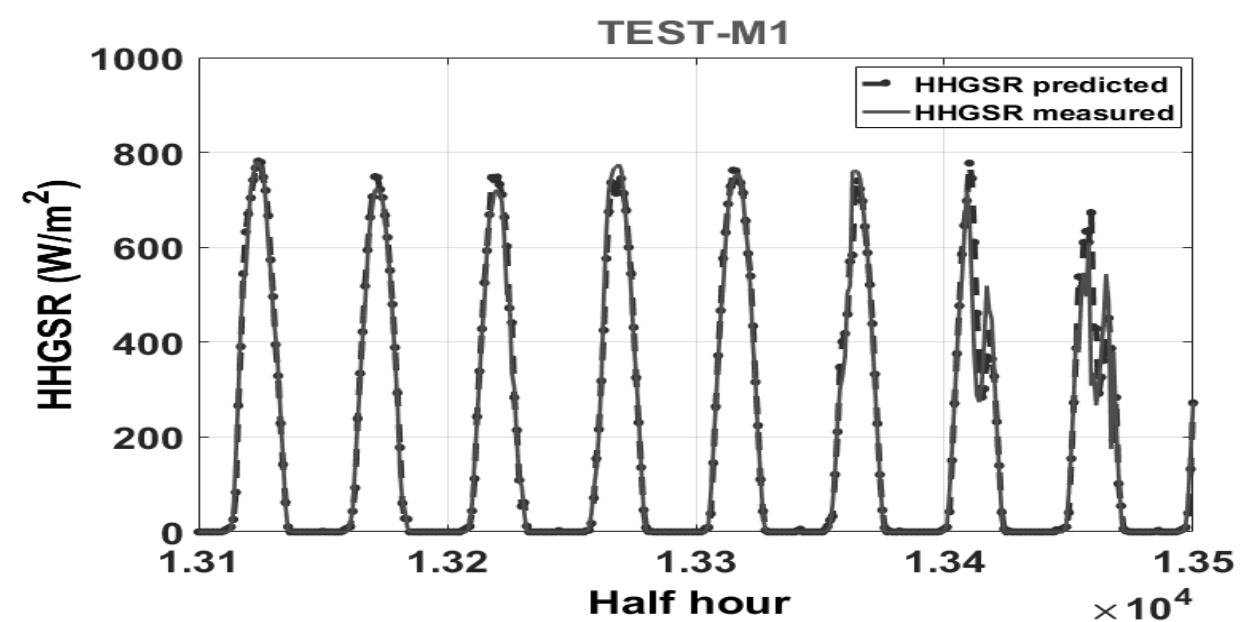

(a)

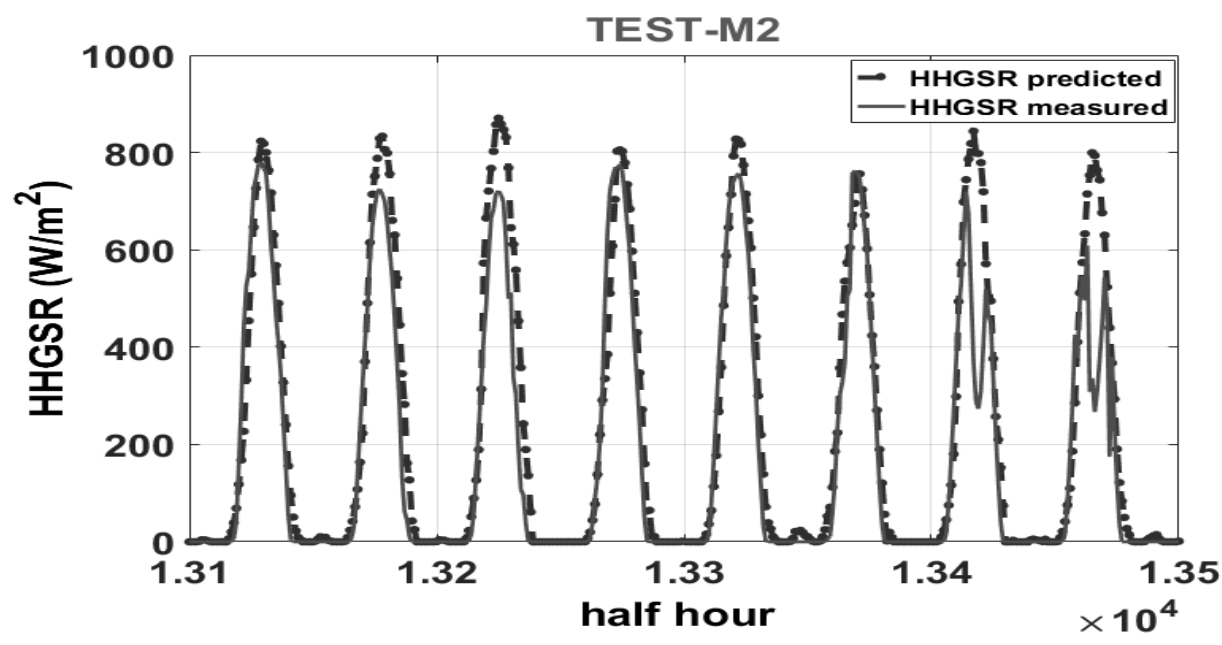

(b)

Figure 11. Evolution of measured and predicted HHGSR times series in the testing phase for (a) M1 model and (b) M2 model.

On the other hand, in Figure 12, the cumulative distributions of measured and predicted HHGSR data were presented. The graphs show clearly the similarity between measured and predicted values for both models except in the winter season for the M2 model. Furthermore, the scattering diagrams (Fig. 13) constitute another means to test the performance and to confirm the precision of the models. Indeed, the obtained values of the correlation coefficients are $\mathrm{R} \_$phase $\mathrm{I}=98.69 \%$ and $\mathrm{R} \_$phase $\mathrm{II}=98.88 \%$ for $\mathrm{M} 1$ model, and $\mathrm{R} \_$phase $\mathrm{I}=94.88 \%$ and $\mathrm{R} \_$phase $\mathrm{II}=94.10 \%$ for M2 model. 


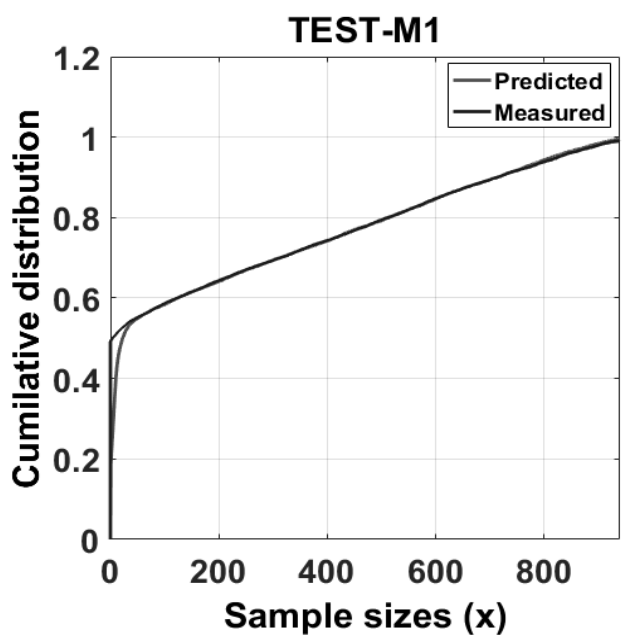

(a)

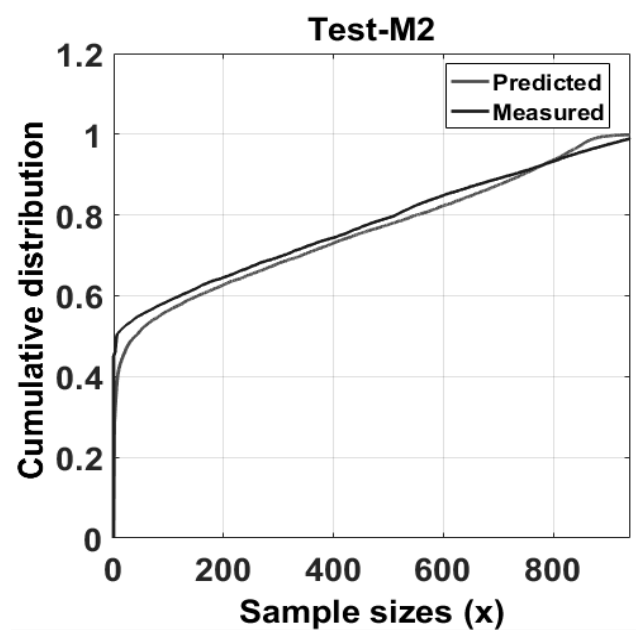

(b)

Figure 12. Cumulative distributions of measured and predicted HHGSR time series for (a) M1 model and (b) M2 model.

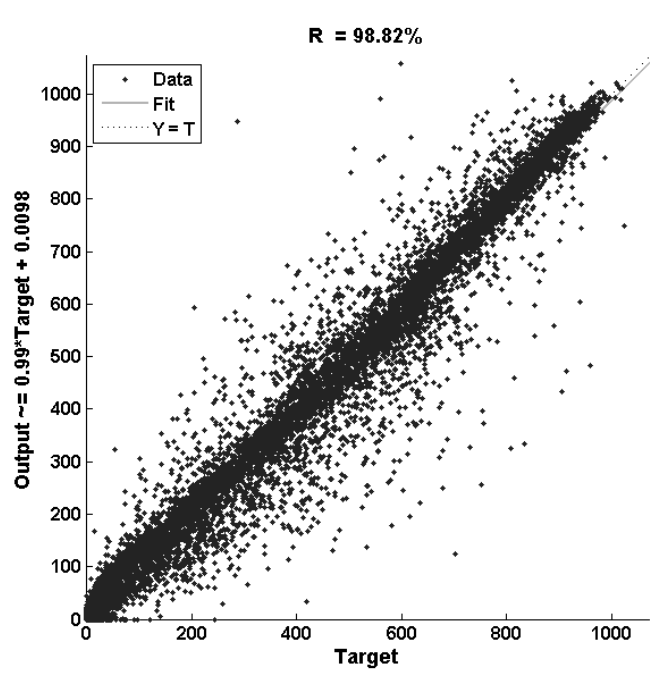

(a)

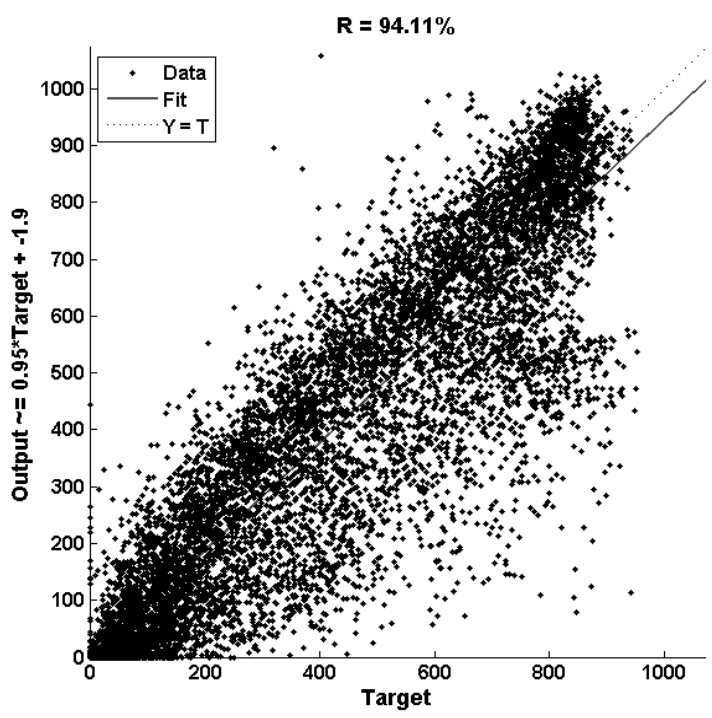

(b)

Figure 13. Scattering diagrams of measured and predicted HHGSR time series for (a) M1 model and (b) M2 model.

From the achieved results, the predicted time series have, in general, the same behavior for both models, and so they are able to accurately perform the prediction of HHGSR, whereas the M1 model is more accurate than the M2 model, due to the use of the autoregressive terms on the direct and the feedback inputs. Consequently, the M1 is the most adequate model to predict the half-hour global solar radiation ahead when a highly accurate prediction is necessary and when the static model M2 cannot precisely predict it. 


\section{CONCLUSION}

The main objective of this paper was to offer a convenient and fast approach to predict the HHGSR values ahead from five parameters (relative humidity, air temperature, wind speed, precipitation, and acquisition time vector in halfhour scale). For this reason, two intelligent models based on the multilayer artificial neural networks are developed. The first one is called multivariate dynamic neural network with feedback connection (M1), which has direct and feedback inputs that are affected by the autoregressive terms. The second one is called multivariate static neural network (M2), which uses direct exogenous inputs. To provide reliable analysis and demonstrate the applicability of the elaborated M1 and M2 models, the used database is divided into two subsets: the first one is used for training and validating the models, and the second one is used for testing the efficiency and the robustness of the models. To evaluate the performances and the precision of the models, the statistical indicators, Centered Mean Square Error, Centered Mean Absolute Error, and correlation coefficient, are used. In terms of these indicators, the developed predictive models can accurately perform the prediction of HHGSR. Besides, the statistical results showed that the M1 model outperforms the M2 model in terms of precision. The obtained values for the M1 model are CMSE $=0.007, \mathrm{CMAE}=0.0401$, and $\mathrm{R}=98.82 \%$ in the testing phase. The achieved results confirm the efficiency of the dynamic model (M1) to accurately predict the HHGSR time series in case of rarity or absence of measurements.

\section{ACKNOWLEDGMENT}

The meteorological database used for this study was funded by the joint international laboratory "Remote Sensing of Water Resources in Semi-Arid Mediterranean Areas".

\section{REFERENCES}

Ahmad, A., T.N. Anderson \& T.T. Lie. 2015. Hourly global solar irradiation forecasting for new zealand. Solar Energy. https:// doi.org/10.1016/j.solener.2015.10.055.

Alsina, Emanuel Federico, Marco Bortolini, Mauro Gamberi \& Alberto Regattieri. 2016. Artificial neural network optimisation for monthly average daily global solar radiation prediction. Energy Conversion and Management, 120: 320-29. https://doi. org/10.1016/j.enconman.2016.04.101.

Assi, Ali H. 2011. Engineering education and research using matlab. https://doi.org/10.5772/1532.

Bear, Glenn W., Haydar J. Al-Shukri \& Albert J. Rudman. 1995. Linear inversion of gravity data for 3-d density distributions. GEOPHYSICS, 60(5): 1354-64. https://doi.org/10.1190/1.1443871.

Bonilla Cardona, Diana A., Nadia Nedjah \& Luiza M. Mourelle. 2017. Online phoneme recognition using multi-layer perceptron networks combined with recurrent non-linear autoregressive neural networks with exogenous inputs. Neurocomputing, 265: 78-90. https://doi.org/10.1016/j.neucom.2016.09.140.

Chabaa, Samira, Abdelouhab Zeroual \& J. Antari. 2010. Identification and prediction of internet traffic using artificial neural networks. Journal of Intelligent Learning Systems and Applications, 02(03): 147-55. https://doi.org/10.4236/jilsa.2010.23018.

Chiteka, K. \& C.C. Enweremadu. 2016. Prediction of Global Horizontal Solar Irradiance in Zimbabwe Using Artificial Neural Networks. Journal of Cleaner Production, 135: 701-11. https://doi.org/10.1016/j.jclepro.2016.06.128.

Dewa, Chandra Kusuma. 2017. Javanese Vowels Sound Classification with Convolutional Neural Network. In Proceeding - 2016 International Seminar on Intelligent Technology and Its Application, ISITIA 2016: Recent Trends in Intelligent Computational Technologies for Sustainable Energy, 123-28. https://doi.org/10.1109/ISITIA.2016.7828645.

EL Badaoui Hicham, Abdelaziz Abdallaoui \& Samira Chabaa. 2013. Using MLP neural networks for predicting global solar radiation. The International Journal of Engineering And Science, 2(12): 48-56.

Gani, A. et al. 2016. Day of the year-based prediction of horizontal global solar radiation by a neural network auto-regressive model. Theoretical and Applied Climatology, 125(3-4): 679-89. https://doi.org/10.1007/s00704-015-1533-8.

Gutierrez-Corea, Federico Vladimir, Miguel Angel Manso-Callejo, Maria Pilar Moreno-Regidor \& Maria Teresa ManriqueSancho. 2016. Forecasting short-term solar irradiance based on artificial neural networks and data from neighboring meteorological stations. Solar energy, 134: 119-31. https://doi.org/10.1016/j.solener.2016.04.020.

Iqdour, R. \& Zeroual, A. 2007. Prediction of daily global solar radiation using fuzzy systems. International Journal of Sustainable Energy, 26: 19-29. 
Iqdour, Radouane \& Abdelouhab Zeroual. 2007. The multi-layered perceptrons neural networks for the prediction of daily solar radiation. International Journal of Signal Processing, 3(1): 24-29.

Jallal, Mohammed Ali, Samira Chabaa, Abdessalam E L Yassini \& Abdelouhab Zeroual. 2019. Air temperature forecasting using artificial neural networks with delayed exogenous input. 2019 international conference on wireless technologies, embedded and intelligent systems (wits), 1-6.

Jallal, Mohammed Ali, Samira Chabaa \& Abdelouhab Zeroual. 2019b. A new artificial multi-neural approach to estimate the hourly global solar radiation in a semi-arid climate site. Theoretical and applied climatology, 139: 1261. https:/doi. org/10.1007/s00704-019-03033-1.

Jallal, Mohammed Ali, Samira Chabaa \& Abdelouhab Zeroual. 2019c. A novel deep neural network based on randomly occurring distributed delayed pso algorithm for monitoring the energy produced by four dual-axis solar trackers. Renewable energy. https://doi.org/10.1016/j.renene.2019.10.117.

Jachner, Stefanie, K Gerald van den Boogaart \& Thomas Petzoldt. 2007. Statistical methods for the qualitative assessment of dynamic models with time delay (r package qualv). Journal of statistical software. https://doi.org/http://dx.doi.org/10.18637/ jss.v022.i08.

Kocadagli, Ozan \& Reza Langari. 2017. Classification of eeg signals for epileptic seizures using hybrid artificial neural networks based wavelet transforms and fuzzy relations. Expert systems with applications, 88: 419-34. https://doi.org/10.1016/j. eswa.2017.07.020.

Le, Xinyi \& Jun Wang. 2014. Robust pole assignment for synthesizing feedback control systems using recurrent neural networks. IEEE transactions on neural networks and learning systems, 25(2): 383-93. https://doi.org/10.1109/TNNLS.2013.2275732.

Li, Fang-fang, Si-ya Wang \& Jia-hua Wei. 2018. Long term rolling prediction model for solar radiation combining empirical mode decomposition (EMD) and artificial neural network (ANN) techniques. https://doi.org/10.1063/1.4999240.

Loutfi, H, A Bernatchou \& R Tadili. 2017. Generation of horizontal hourly global solar radiation from exogenous variables using an artificial neural network in Fes (morocco). International Journal of Renewable Energy Research, 7(3).

Min, Zeng \& Lin Cao. 2017. Application of the neural network in diagnosis of breast cancer based on Levenberg-Marquardt algorithm, 268-272.

Mohandes, M., S. Rehman \& T.O. Halawani. 1998. Estimation of global solar radiation using artificial neural networks. Renewable energy, 14(1): 179-84. https://doi.org/10.1016/S0960-1481(98)00065-2.

Muhammad, Syed, Aqil Burney, Tahseen Ahmed Jilani \& Cemal Ardil. 2008. Levenberg-Marquardt algorithm for Karachi stock exchange share rates forecasting. Computational Intelligence, 1(2): 168-73.

Prabhaker Reddy, Ginuga, G. Radhika \& K Anil. 2012. Control of continuous stirred tank reactor using artificial neural networks based predictive control. Advanced Materials Research, 550-553: 2908-12. https://doi.org/10.4028/www.scientific. net/AMR.550-553.2908.

Rzadkowski, Romuald, Krzysztof Dominiczak, Wojciech Radulski \& R. Szczepanik. 2015. Thermoelastic steam turbine rotor control based on neural network. In Journal of Physics: Conference Series. Vol. 662. https://doi.org/10.1088/17426596/662/1/012021.

Safi, S., A. Zeroual \& M.M. Hassani. 2002. Modelling solar half-hour data using fourth order cumulants. International Journal of Solar Energy. https://doi.org/10.1080/01425910214357.

Saha, Sajib, Fan Gu, D Ph, A M Asce, Xue Luo, D Ph, A M Asce, Robert L Lytton, D Ph \& F Asce. 2018. Prediction of soilwater characteristic curve for unbound material using Fredlund - Xing equation-based ANN approach 30(5): 1-10. https:// doi.org/10.1061/(ASCE)MT.1943-5533.0002241.

Shaddel, Mehdi, Dawood Seyed Javan \& Parisa Baghernia. 2016. Estimation of hourly global solar irradiation on tilted absorbers from horizontal one using artificial neural network for case study of Mashhad. Renewable and Sustainable Energy Reviews, 53: 59-67. https://doi.org/10.1016/j.rser.2015.08.023.

Sheng Li, Lian, Sheng jiang Gan \& Xiang dong Yin. 2017. Feedback recurrent neural network-based embedded vector and its application in topic model. Eurasip Journal on Embedded Systems, 2017(1). https://doi.org/10.1186/s13639-016-0038-6.

Zeroual, A., Ankrim, M. \& Wilkinson, A.J. 1995. Stochastic modelling of daily global solar radiation measured in Marrakesh, Morocco. Renew. Energy 6: 787-793.

Zeroual, A., Ankrim, M. \& Wilkinson, A.J. 1996. The diffuse-global correlation : Its application to estimating solar radiation on tilted surfaces in Marrakesh, Morocco. Renew. Energy, 7: 1-13. 